\title{
E se a superfície for áspera? Um estudo sobre a influência da força de atrito em colisões inelásticas através de videoanálise
}

What if the surface is rough? A study on the influence of the friction force in inelastic collisions through video analysis

\author{
R.S. Franco ${ }^{1}$, V.S. Miranda ${ }^{1}$, R.S. Dutra ${ }^{2} @$, L.C. Ribeiro ${ }^{1}$ \\ ${ }^{1}$ Centro Federal de Educação Tecnológica Celso Suckow da Fonseca, Nova Iguaçu, RJ, Brasil. \\ ${ }^{2}$ Instituto Federal do Rio de Janeiro, Laboratório de Instrumentação e Simulação Computacional, Paracambi, RJ, Brasil.
}

Recebido em 31 de dezembro de 2020. Revisado em 19 de março de 2021. Aceito em 19 de abril de 2021.

\begin{abstract}
Este trabalho apresenta um estudo que explica a física associada ao conjunto das sucessivas colisões inelásticas de um sólido esférico contra a superfície rígida do piso do laboratório, após ser lançado de uma determinada altura. O centro de massa do sólido é rastreado a partir da videoanálise realizada através do conhecido software educacional Tracker, possibilitando a obtenção da velocidade de seu centro de massa durante todo o caminho percorrido. O momento linear do sólido é obtido nos instantes imediatamente antes e após as colisões, permitindo o cálculo de sua dissipação, assim como da dissipação da energia cinética, durante esses eventos. O papel desempenhado pela força de atrito durante as colisões também é avaliado, através de uma grandeza pouco conhecida na literatura de física básica, designada por coeficiente de restituição tangencial. Medidas dessa grandeza apresentaram valores próximos à unidade e, portanto, revelaram quantitativamente que, para o experimento realizado, é ínfima a contribuição da força de atrito para o impulso durante as colisões, se comparada à fornecida pela força normal, também exercida pelo piso do laboratório. Esse resultado é implicitamente considerado em modelos de colisões apresentados em livros didáticos de física básica, onde, sem nenhuma justificativa, a força de atrito é ignorada.
\end{abstract}

Palavras-chave: Colisões inelásticas, videoanálise, coeficiente de restituição tangencial.

This paper presents a study that explains the physics associated to the set of successive inelastic collisions of a spherical solid against the rigid surface of the lab's floor after being launched from a certain height. The solid's center of mass is tracked by means of a video analysis through the well-known educational software Tracker, allowing us to obtain its velocity in the whole described path. The solid's linear momentum is then obtained for the instants immediately before and after the collisions, permitting to evaluate its dissipation as well as the kinetic energy dissipation during these events. The role played by the friction force during the collisions is also evaluated through a not widely known quantity in basic physics literature, named by tangential restitution coefficient. Measurements of this quantity presented values close to unit and, therefore, quantitatively revealed that, to the performed experiment, the frictional force contribution to the impulse during the collisions are tiny if compared to that provided by the normal force applied by the lab's floor. This result is implicitly considered in the collisions models presented in the basic physics' textbooks, where, without any justification, the frictional force is ignored. Keywords: Inelastic collision, video analysis, tangential restitution coefficient.

\section{Introdução}

O fenômeno da colisão é observado com frequência na natureza e manifesta-se desde eventos mais cotidianos, como o cair de um objeto sobre uma superfície, ou o choque entre duas esferas rígidas durante um jogo de bilhar, a eventos mais complexos, que necessitam de equipamentos sofisticados para serem analisados, como é o caso das colisões entre partículas subatômicas produzidas em aceleradores de altas energias, geralmente em projetos que têm por objetivo investigar a

\footnotetext{
*Endereço de correspondência: rafael.dutra@ifrj.edu.br
}

possível existência de constituintes elementares dessas partículas e a natureza das forças que mantém coesas suas estruturas [1].

Nos cursos de física básica 24 4 o estudo das colisões é geralmente apresentado com base nos princípios de conservação do momento linear e da energia cinética total do sistema, esse último com validade restrita ao caso específico das colisões perfeitamente elásticas, precisando, no caso das colisões inelásticas, ser adaptado com o auxílio de um coeficiente de restituição que quantifica a energia dissipada [5]. No infinitésimo de tempo que corresponde à duração da colisão entre dois ou mais corpos [6], ocorre uma interação entre eles, que 
se manifesta pela atuação recíproca de uma ou mais forças, responsáveis por alterar seus momentos lineares. Se o sistema estiver isolado, ou seja, se inexistirem forças externas atuando sobre seus componentes, o momento linear total é conservado. Quanto à variação da energia cinética total, dependerá do grau de elasticidade da colisão, podendo ser avaliada através do coeficiente de restituição mencionado anteriormente.

Neste artigo consideramos um "sistema" não isolado, composto apenas por um corpo sólido, de massa $m$ e velocidade inicial de módulo $v_{0}$, que tem seu momento linear e energia cinética alterados após colidir de forma oblíqua com a superfície plana e rígida do piso do laboratório. Durante a colisão, duas forças atuam sobre esse corpo, a saber, a força normal à superfície do piso e a força de atrito, ambas, em alguma proporção, contribuindo para o impulso aplicado e para a consequente variação do momento linear e da energia cinética do sólido. Em relação a essa última, sua variação é analisada a partir do coeficiente de restituição, que, neste artigo, será designado por coeficiente de restituição normal, explicitando a contribuição predominante da força normal de contato para o impulso sobre o sólido em relação à contribuição dada pela força de atrito. A pertinência de tal especificação encontra justificativa em um contexto de discussões geralmente ausentes nos livros didáticos [7 7 ] e que estão relacionadas à contribuição efetiva das forças de atrito para a variação do momento linear e da energia cinética do sólido. Tais discussões são levantadas neste artigo com base no estudo da atuação de cada uma dessas forças e da quantificação de seus efeitos, obtida a partir de medidas experimentais realizadas com base no monitoramento das colisões através da técnica de videoanálise.

Com relação à colisão descrita no parágrafo anterior, a Figura 1 mostra um diagrama onde são indicadas as forças peso e normal que atuam sobre o corpo no infinitésimo de tempo que corresponde a esse evento. Diagrama esse que corresponde ao modelo geralmente considerado nos livros didáticos, que desconsidera a atuação da força de atrito tangente à superfície do piso. Esse diagrama também indica os ângulos de incidência $\theta_{A}$ e de reflexão $\theta_{D}$, além das velocidades de módulos $v_{A}$ e $v_{D}$ consideradas nos instantes imediatamente antes e depois da colisão. Na maioria das vezes os autores consideram uma reflexão especular em que $\theta_{D}=\theta_{A}$, como indicado na Figura 1(a). Esse tipo de colisão ocorre quando o coeficiente de restituição normal, designado pela letra $e$, é igual a unidade, $e=1$, caracterizando assim uma colisão perfeitamente elástica. Nesse caso o momento linear do corpo sofre uma reflexão, preservando seu módulo, e, em consequência, a energia cinética do corpo. No caso da reflexão não especular, mostrada na Figura 1(b), temos um coeficiente de restituição menor que a unidade, $e<1$, indicando uma dissipação de energia cinética em decorrência da variação do módulo da componente vertical $y$ do vetor momento linear,

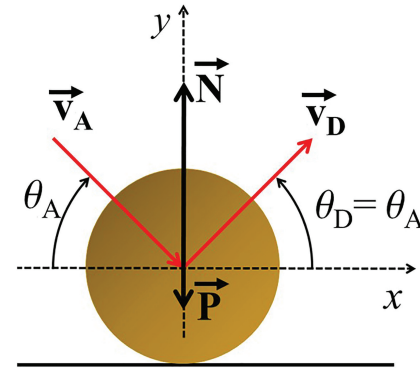

(a) (b)

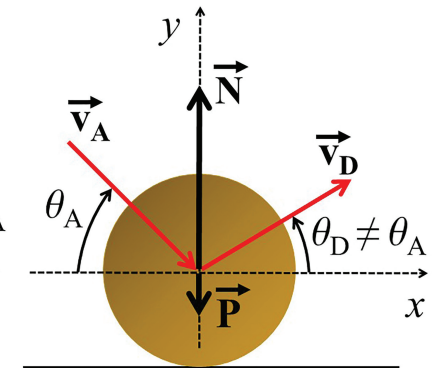

Figura 1: Colisão bidimensional de uma esfera com o solo. (a) Colisão elástica com coeficiente de restituição normal $e=1$. (b) Colisão inelástica com coeficiente de restituição normal $e<1$.

perpendicular à superfície do solo. Já a componente $x$ do momento linear, tangencial a essa superfície, é mantida constante. Nesse caso temos $\theta_{D}<\theta_{A}$, conforme indicado na figura.

Em ambas as situações descritas no parágrafo anterior e ilustradas na figura 1, a discussão acerca da rugosidade da superfície na qual a colisão ocorre é geralmente omitida nos livros didáticos de física básica [10]12], que deixam implícita a ideia de que essa superfície é lisa ao ponto da força de atrito poder ser desprezada durante a colisão. E se essa superfície for áspera, o quão significativo seria o seu efeito, a ponto de termos que considerar a força de atrito no modelo da colisão? Para responder a essa pergunta, investigaremos a contribuição da força de atrito para a variação do vetor momento linear da partícula durante as colisões, verificando se a componente horizontal desse vetor sofre ou não variações significativas em decorrência desses eventos. Nesse contexto, apresentamos um estudo experimental das colisões através do monitoramento do movimento do sólido por videoanálise, onde o efeito impulsivo da força de atrito nas colisões é mensurado por meio de uma grandeza conhecida como coeficiente de restituição tangencial e designada pela letra $\beta$ [13], grandeza essa muito pouco discutida na literatura de física básica, como mencionado anteriormente.

O surgimento de softwares de videoanálise [14] tem acarretado uma crescente expansão de trabalhos acadêmicos destinados ao desenvolvimento de técnicas didáticas voltadas ao aprendizado de determinados tópicos da disciplina de física, entre os quais destacamos os que envolvem problemas relacionados aos sistemas mecânicos abordados no ensino médio e nos primeiros períodos dos cursos de graduação das carreiras de ciências exatas. Tais problemas envolvem desde aplicações esportivas [15], passando por problemas clássicos de queda livre [16, 17] e de dinâmica de rotação de sólidos [18 20], até a análise de colisões, que é o tema objeto de estudo deste artigo. O uso das Tecnologias da Informação e Computação (TICs) vem sendo progressivamente incorporado ao ensino de física [21, 22, 
legitimando, nos últimos anos, uma área de pesquisa em ensino de física com ênfase na modelagem computacional por meio de simulações computacionais 23. e de videoanálise educacional [24]. Do ponto de vista epistemológico, a utilização das TICs permitem trazer para a sala de aula a discussão acerca de como os modelos físicos são pensados e concebidos [25], bem como os seus limites de validade, promovendo assim um ensino de física mais participativo e com maior enfoque no desenvolvimento do senso crítico dos alunos, colocandoos na posição de protagonistas do processo ensino e aprendizagem.

Neste trabalho utilizamos o software livre Tracker para monitorar o movimento do corpo associado ao problema clássico de colisão descrito nos parágrafos anteriores, ilustrado na Figura 2(a) e que consiste em um corpo colidindo sucessivas vezes com a superfície rígida do piso do laboratório após abandonar, com velocidade horizontal de módulo $v_{0}$, a base de um perfil curvo, localizada a uma altura $h$, medida em relação a esse piso. Inserimos o nosso trabalho no contexto da modelagem computacional voltada para o ensino de física, onde, por meio dos resultados da videoanálise, discutimos a plausabilidade de se considerar a conservação da componente horizontal do momento linear em uma colisão oblíqua contra uma superfície áspera.

Por fim, este trabalho encontra-se dividido com a segunda seção dedicada à descrição do experimento realizado, a terceira seção dedicada à apresentação e desenvolvimento do modelo teórico adotado, a quarta seção à análise e discussão dos resultados obtidos, a quinta, às conclusões e considerações finais, e a sexta, aos agradecimentos.

\section{Descrição do Experimento e Monitoramento por Videoanálise}

O experimento realizado tem por objetivo investigar a física que governa as colisões de um pequeno sólido esférico com a superfície horizontal rígida do piso do laboratório e encontra-se ilustrado na Figura 2(a), que mostra uma fotografia estroboscópica indicando a trajetória percorrida por esse sólido no período de tempo contado a partir do instante em que ele abandona, com velocidade horizontal de módulo $v_{0}$, a base de uma rampa que é utilizada para acelerá-lo. Essa base marca a posição do lançamento horizontal e encontrase a uma altura $H_{0}$, medida em relação ao solo. Após o lançamento, o sólido segue perdendo altura, devido a atuação da força peso, até colidir obliquamente com a superfície rígida do piso do laboratório, com velocidade de módulo $v_{A}$ e ângulo de incidência $\theta_{A}$, momento em que é impulsionado pelas forças a ele aplicadas por essa superfície, sendo desviado com uma velocidade de módulo $v_{D}$, que forma um ângulo $\theta_{D}$ com a horizontal. Em seguida, o sólido volta a subir, atingindo uma nova altura $H_{1}<H_{0}$ em um determinado instante, a partir do qual volta a perder altura, até sofrer uma segunda colisão. Esse processo se repete com o sólido sofrendo sucessivas colisões e atingindo determinadas alturas máximas, sempre com $H_{n}<H_{n-1}$, onde $H_{n}$ indica a altura máxima atingida após a enésima colisão, sendo, no caso do experimento realizado, $n=1,2,3$ e 4 . Além dessas, a altura $H_{0}$, correspondente à posição do lançamento inicial, também é de grande importância para o cálculo da energia mecânica dissipada nas sucessivas colisões, como será visto na seção a seguir.

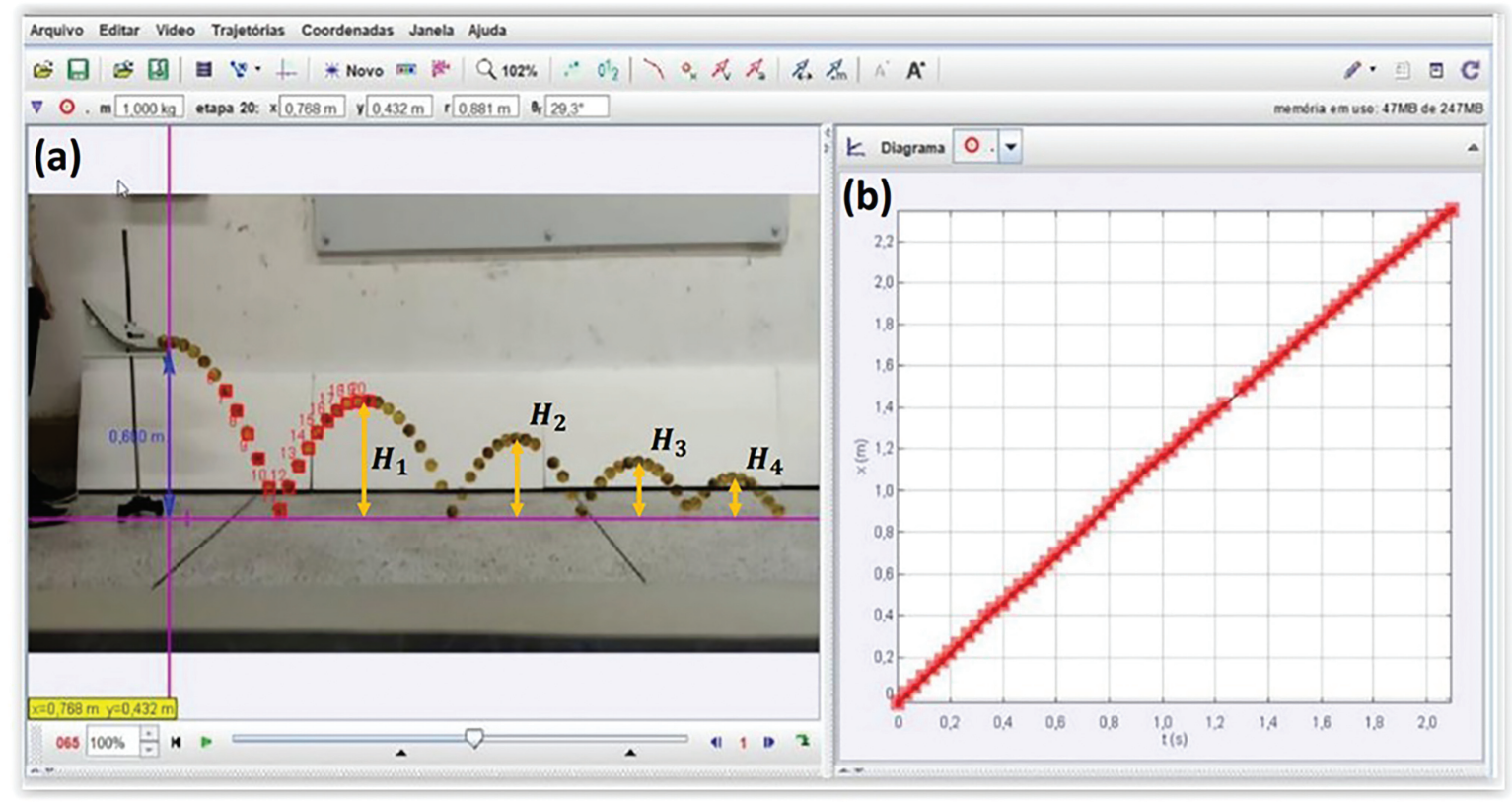

Figura 2: (a) Fotografia estroboscópica do movimento da esfera, obtida a partir da videoanálise realizada utilizando o Tracker. (b) Coordenada horizontal do centro de massa da esfera em função do tempo. 
A perda gradativa de energia mecânica é indicada na relação $H_{n}<H_{n-1}$ entre as sucessivas alturas atingidas pelo sólido e está relacionada ao grau de elasticidade das colisões, que, como mencionado na introdução, pode ser quantificado através de um coeficiente cujo valor numérico está relacionado ao material que constitui o piso do laboratório e o próprio sólido lançado. No caso, o experimento descrito foi realizado sobre a superfície rígida de um chão de cimento e o sólido lançado consiste em uma pequena esfera de borracha. A análise desse experimento tem por base o monitoramento do centro de massa dessa esfera por meio de videoanálise realizada com o software Tracker (versão 5.1.3) [14]. O vídeo analisado foi produzido a partir de uma câmera $16 \mathrm{Mp}+12 \mathrm{Mp}$ de um celular Xiaomi Modelo Mi 9 com capacidade de captura de 30 fps (frames por segundo). O Tracker opera a partir de marcações realizadas manualmente com o cursor do mouse nas posições percorridas pelo centro de massa do sólido lançado, identificadas através da sequência de imagens do vídeo analisado. A fim de quantificar de forma correta as grandezas físicas fornecidas, como posição, velocidade e aceleração do sólido, o Tracker precisa ser calibrado com a definição da distância real entre dois pontos quaisquer de um determinado frame. Com isso ele reescalona as distâncias em cada frame e, com o rastreamento da posição da esfera no decorrer do vídeo, consegue atribuir valores reais para as grandezas físicas mencionadas. No experimento foi utilizado como referência a altura da base da canaleta, medida em relação ao piso do laboratório. Essa altura é de $60 \mathrm{~cm}$, conforme indicado na Figura 2(a).

A Figura 2(b) mostra o gráfico obtido através da videoanálise para a componente horizontal $x$ da posição do centro de massa da esfera em função do tempo. A dependência temporal praticamente linear dessa componente indica que a componente horizontal da velocidade do centro de massa da esfera, $v_{x}$, permanece aproximadamente constante durante o movimento, o que conduz, em um primeiro momento, à conclusão de que são mínimos os efeitos produzidos por forças resistivas horizontais que eventualmente atuam sobre esse sólido, tais como a componente horizontal da força associada à resistência do ar e a própria força de atrito aplicada pela superfície durante as colisões. Em relação à força de atrito, cabe mencionar a relevância de sua atuação sobre o sólido em circunstâncias específicas, motivo pelo qual essa força encontra-se incorporada ao modelo de colisão apresentado na seção a seguir, sendo os efeitos provenientes de sua atuação sobre o sólido estimados a partir dos resultados experimentais extraídos por videoanálise.

\section{Modelo Teórico}

Esta seção é dedicada ao estudo da física que governa as colisões entre o sólido lançado e o piso do laboratório,

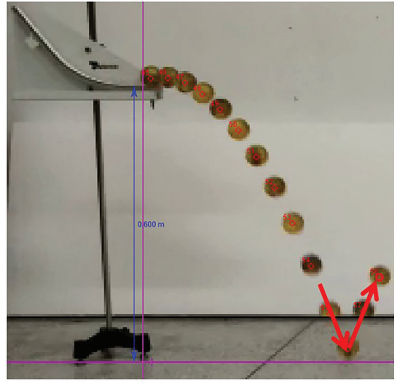

(a)

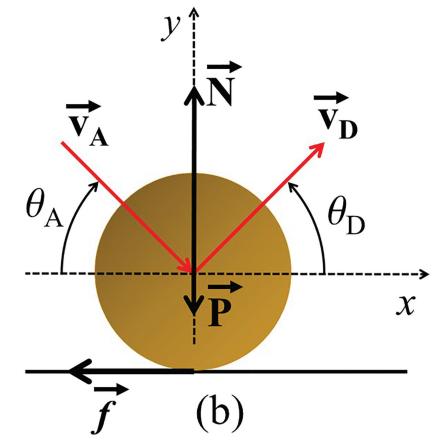

Figura 3: (a) Fotografia estroboscópica mostrando o instante em que a esfera colide com o solo pela primeira vez. (b) Diagrama mostrando as forças que atuam sobre a esfera durante as colisões com o solo.

tendo por base a proposição de um modelo que incorpora as grandezas físicas relacionadas a esses eventos. Tal modelo encontra-se representado na Figura 3 , que mostra em (a) uma fotografia da primeira colisão e em (b) um diagrama indicando as grandezas físicas a ela associadas. Em particular, os vetores em vermelho representam as velocidades de módulos $v_{A}$ e $v_{D}$ nos instantes imediatamente antes e depois da colisão, respectivamente. Nessa figura também são indicados os ângulos de incidência $\theta_{A}$ e de reflexão $\theta_{D}$, assim como as forças peso, $\vec{P}$, normal, $\vec{N}$, e de atrito, $\vec{f}$, que atuam sobre o sólido, sendo a atuação das duas últimas restrita ao intervalo de tempo infinitesimal, da ordem de $1 \mathrm{~ms}$, correspondente à duração da colisão [6]. Durante esse infinitésimo de tempo, a força normal de contato $\vec{N}$ e a força de atrito $\vec{f}$ são responsáveis por alterar a velocidade do centro de massa do sólido e podem ser entendidas, respectivamente, como as componentes normal e tangencial da força de contato aplicada ao sólido pelo piso do laboratório. Para quantificar essa alteração utilizamos os coeficientes de restituição definidos ao longo das direções normal e tangencial, e designados pelas letras e e $\beta$, respectivamente. Pensando em aplicar a análise desse problema em uma classe de ensino médio, faremos aqui uma abordagem mais simplificada, na qual desprezaremos a velocidade de rotação da esfera, tratando-a como uma partícula. Essa aproximação é válida, desde que a velocidade tangencial, no momento da colisão, em um ponto da borda da esfera, possa ser desprezada frente a velocidade do centro de massa. Assim, as velocidades relativas de aproximação e afastamento em relação ao solo serão as mesmas para o centro de massa da esfera e para um ponto qualquer localizado em sua superfície. No caso, o coeficiente de restituição normal $e$ aparece com frequência nos livros didáticos convencionais, sendo definido pela razão entre as componentes verticais das velocidades de afastamento (depois da colisão) e de aproximação (antes da colisão) do centro de massa do sólido, medidas em relação a um observador ligado ao piso do laboratório. Sendo essas 
componentes designadas, respectivamente, por $v_{A, y} \mathrm{e}$ $v_{D, y}$, obtêm-se, para o coeficiente de restituição normal,

$$
e=-\frac{v_{D, y}}{v_{A, y}}
$$

Desprezada a resistência do ar, o que é razoável, se a esfera lançada não for por demasiado leve, essas componentes também se relacionam com as alturas máximas, $H_{A}$ e $H_{D}$, entre duas colisões sucessivas, sendo $v_{A, y}=-\sqrt{2 g H_{A}}$ e $v_{D, y}=\sqrt{2 g H_{D}}$, que, substituídas na equação (1), fornecem

$$
e=\sqrt{\frac{H_{D}}{H_{A}}}
$$

A equação (2) pode ser generalizada de modo a considerar a enésima colisão com o piso do laboratório, resultando na expressão

$$
e=\sqrt{H_{n} / H_{n-1}},
$$

onde $H_{n-1}$ e $H_{n}$ são, respectivamente, as alturas máximas atingidas pelo sólido antes e depois dessa colisão. Por meio da equação (3) temos as seguintes relações para as alturas máximas consecutivas atingidas após cada umas das colisões: $H_{1}=H_{0} e^{2}, H_{2}=H_{1} e^{2}$, $H_{3}=H_{2} e^{2}, \ldots, H_{n}=H_{n-1} e^{2}$, a partir das quais, obtêmse, por recursividade,

$$
H_{n}=H_{0} e^{2 n},
$$

que estabelece a relação entre o coeficiente de restituição normal $e$, a altura máxima atingida após a enésima colisão, $H_{n}$, e a altura $H_{0}$ do ponto de lançamento horizontal localizado na base da rampa.

$O$ coeficiente de restituição tangencial $\beta$ associado à enésima colisão é definido pela relação entre as componentes horizontais das velocidades relativas de afastamento, $v_{n, x}$, e de aproximação, $v_{n-1, x}$, do centro de massa do sólido, medidas em relação ao ponto de colisão [26],

$$
\beta=\frac{v_{n, x}}{v_{n-1, x}}
$$

Essa equação é válida na hipótese de não deslizamento entre as superfícies da esfera e do piso, ou seja, se, durante o contato, a magnitude da força de atrito estático, $f$, obedecer à restrição $f \leq \mu_{e} N$, sendo $\mu_{e}$ o coeficiente de atrito estático entre as superfícies envolvidas e $N$ a força normal de contato. A adoção de tal hipótese é razoável no experimento descrito neste artigo, tendo em conta os baixos valores que foram obtidos para a componente horizontal da aceleração, resultante da atuação da força $\vec{f}$, como indicado no resultado da Figura 2 (b), que mostra o comportamento aproximadamente linear da coordenada $x$ da posição do centro de massa da esfera em função do tempo. Utilizando a equação (5) temos as seguintes relações para as componentes horizontais da velocidade, entre colisões sucessivas: $v_{1, x}=v_{0, x} \beta$, $v_{2, x}=v_{1, x} \beta, v_{3, x}=v_{2, x} \beta, \ldots, v_{n, x}=v_{n-1, x} \beta$. Por meio das equações anteriores, obtêm-se, por recursividade, a expressão

$$
v_{n, x}=v_{0, x} \beta^{n},
$$

que estabelece uma relação entre o coeficiente de restituição tangencial $\beta$, a componente horizontal $v_{n, x}$ da velocidade do centro de massa da esfera imediatamente após a enésima colisão e a velocidade horizontal $v_{0}=v_{0, x}$ do centro de massa da esfera no instante em que ela abandona a canaleta.

Uma grandeza importante a ser considerada no estudo das colisões é o impulso $\vec{J}$ proveniente da resultante $\vec{F}(t)$ das forças aplicadas à esfera durante as colisões, grandeza essa que, de acordo com o teorema do impulso, está associada à variação do momento linear $\vec{P}$ dessa esfera, $\Delta \vec{P}=m \overrightarrow{\mathrm{v}}_{D}-m \overrightarrow{\mathrm{v}}_{A}$, através da equação

$$
\vec{J}=\lim _{t \rightarrow t_{0}} \int_{t_{0}}^{t} \vec{F}(t) d t=m \overrightarrow{\mathrm{v}}_{D}-m \overrightarrow{\mathrm{v}}_{A},
$$

em que o limite $t \longrightarrow t_{0}$ indica a magnitude ínfima das escalas de tempo associadas à duração das colisões. A análise das forças que atuam sobre a esfera durante essas colisões, de acordo com o modelo apresentado na Figura 3(a), conduz à conclusão de que a componente do impulso normal à superfície do piso é proveniente da força normal aplicada à esfera durante o contato. Essa componente, correspondente à enésima colisão, é designada por $J_{N, n}$ e, de acordo com a equação acima, está associada à variação da componente vertical do momento linear da esfera através da equação $J_{N, n}=$ $m v_{n, y}-m v_{n-1, y}$, sendo $v_{n-1, y}$ e $v_{n, y}$, respectivamente, as componentes verticais das velocidades relativas de aproximação e de afastamento do centro de massa da esfera, medidas em relação ao ponto onde a colisão ocorre. Como a energia mecânica é conservada no intervalo entre duas colisões sucessivas, essas componentes podem ser escritas como $v_{n-1, y}=-\sqrt{2 g H_{n-1}}$ e $v_{n, y}=\sqrt{2 g H_{n}}$, sendo essa hipótese de conservação, entre duas colisões sucessivas, válida desde que a resistência do ar possa ser desprezada 11 Substituindo essas velocidades na expressão de $J_{N, n}$ apresentada acima, chegamos à relação $J_{N, n}=m \sqrt{2 g H_{n}}+m \sqrt{2 g H_{n-1}}$. Assim, utilizando a equação (4), obtêm-se que a componente normal do impulso exercido pelo piso do laboratório durante a enésima colisão é expressa em termos do coeficiente de restituição normal $e$, como

$$
J_{N, n}=m \sqrt{2 g H_{0}}\left(\frac{1+e}{e}\right) e^{n} .
$$

\footnotetext{
1 No caso, o comportamento aproximadamente linear do gráfico $x$ versus $t$ na Figura 2(b) e o valor obtido para a aceleração da gravidade local, na seção 4, próximo do experimentalmente aceito, de $9,81 \mathrm{~m} / \mathrm{s}^{2}$, considerando as incertezas experimentais, justificam essa hipótese.
} 
No caso da componente tangencial do impulso produzido pela atuação da força de atrito durante a colisão considerada, a equação (7) fornece que essa componente, designada por $J_{T, n}$, corresponde à variação da componente horizontal do momento linear da esfera, sendo escrita como $J_{T, n}=m v_{n, x}-m v_{n-1, x}$, com $v_{n-1, x}$ e $v_{n, x}$ correspondendo, respectivamente, às componentes horizontais do vetor velocidade nos instantes imediatamente antes e depois da colisão. Assim, considerando conjuntamente a equação (6), obtêm-se

$$
J_{T, n}=-m v_{0, x}\left(\frac{1-\beta}{\beta}\right) \beta^{n},
$$

que relaciona o impulso associado à enésima colisão, a velocidade inicial de lançamento $v_{0, x}$ e o coeficiente de restituição tangencial $\beta$. A partir das equações (8) e (9) e dos resultados obtidos através da videoanálise, investigamos o papel desempenhado por cada uma das componentes da força de contato durante as colisões oblíquas. Inicialmente, através das equações (5), podese concluir que, se a componente tangencial do vetor velocidade do centro de massa da esfera se mantiver aproximadamente constante durante as colisões, como indicado pela dependência temporal aproximadamente linear da componente $x$ do vetor posição apresentada no resultado da Figura 2(b), o coeficiente de restituição tangencial deverá assumir valores próximos à unidade, $\beta \approx 1,0$, resultando, de acordo com a equação $(9)$, em um impulso tangencial praticamente nulo, $J_{T, n} \approx 0$. Esse resultado será demostrado experimentalmente na seção a seguir, onde será estabelecido de forma quantitativa que o impulso tangencial é praticamente nulo durante as colisões, $J_{T, n} \approx 0$, motivo pelo qual é sumariamente desprezado nos livros de física básica.

\section{Análise e Discussão dos Resultados}

A análise de vídeo do movimento descrito pelo sólido, considerado a partir do instante em que ele abandona a rampa com velocidade horizontal de módulo $v_{0}$, e passando pelas sucessivas colisões com o piso do laboratório, como apresentado na Figura 2(a), permitiu a obtenção de grandezas físicas a ele associadas, extraídas de forma direta a partir de análise gráfica, a exemplo da componente horizontal da velocidade do centro de massa, dada pela inclinação da reta obtida para a componente horizontal da posição em função do tempo, apresentada na Figura 2(b). Esse resultado é de fundamental importância e está fortemente relacionado ao objetivo deste artigo, pois assinala a relevância mínima atribuída a atuação da força horizontal de atrito durante as colisões, corroborando assim com o modelo frequentemente apresentado nos livros didáticos. Tal constatação é discutida de forma quantitativa nesta seção, através de medidas obtidas para os coeficientes de restituição tangencial $\beta$ associados às colisões.

Uma das grandezas físicas obtidas a partir da análise gráfica do movimento é a velocidade da esfera nos instantes imediatamente após as colisões, composta, no caso, por uma componente horizontal $v_{0 x}=v_{x}$, extraída a partir do gráfico da Figura 2(b), e uma componente vertical, $v_{0 y}$, obtida a partir dos gráficos apresentados na Figura 4, que mostram a dependência temporal da coordenada $y$ do vetor posição do sólido nos intervalos de tempo (a) entre o lançamento inicial e a primeira colisão e (b, c e d) entre as colisões sucessivas, até a quarta colisão $(n=4)$. Os resultados apresentados na Figura 4 ilustram a dependência quadrática da componente $y$ do vetor posição em função do tempo, descrita por $y(t)=y_{0}+v_{0 y} t-\frac{1}{2} g t^{2}$, sendo $y_{0}$ e $v_{0 y}$ os valores iniciais das componentes $y$ dos vetores posição e velocidade, respectivamente, e $g$, o módulo da aceleração da gravidade local. Com o objetivo de analisar esses resultados de forma quantitativa, utilizamos o próprio Tracker para ajustar os pontos experimentais obtidos com uma parábola dada por $y(t)=A t^{2}+B t+C$, cujos coeficientes são identificados com as grandezas físicas correspondentes, sendo $A=-g / 2, B=v_{0 y} \mathrm{e}$ $C=y_{0}$. Os valores dos parâmetros extraídos a partir desse ajuste $(A, B$ e $C$ ) são mostrados nos insets dos painéis da Figura 4 e estão em unidades do Sistema Internacional (SI). Do painel (a) podemos extrair a altura e a velocidade horizontal de lançamento, dada por $y_{0}=C=63,3 \mathrm{~cm}\left(\delta y_{0}=0,6 \mathrm{~cm}\right)$. Observa-se que essa altura corresponde à altura da canaleta, de $60 \mathrm{~cm}$, mais a medida do raio da esfera, de $2,2 \mathrm{~cm}$, com uma pequena diferença que pode ser justificada por erros associados ao lançamento do sólido, como, por exemplo, ao fato de não ser perfeitamente horizontal, dado o pequeno valor da velocidade extraída da videoanálise, de $v_{0 y}=B=-0,29 \mathrm{~m} / \mathrm{s}\left(\delta v_{0 y}=0,09 \mathrm{~m} / \mathrm{s}\right)$, ou a uma pequena imprecisão na captura do plano em que o movimento ocorre. As incertezas associadas a essas grandezas, apresentadas entre parênteses, foram obtidas com o software SciDAVis [27]. A partir dos valores extraídos para a aceleração da gravidade local $g \mathrm{em}$ cada um dos painéis, através da relação $g=-2 A$, obtivemos um valor médio para essa grandeza, dado por $\bar{g}=9,7 \mathrm{~m} / \mathrm{s}^{2}$, com um erro de $\delta \bar{g}=0,3 \mathrm{~m} / \mathrm{s}^{2}$, estimado a partir da diferença $\left(g^{\text {máx }}-g^{\text {min }}\right) / 2$, sendo $g^{\text {máx }}$ e $g^{\text {min }}$, respectivamente, os valores máximo e mínimo de $g$, obtidos com os parâmetros extraídos a partir dos resultados apresentados nos painéis correspondentes. Esse erro representa um desvio percentual de aproximadamente $3 \%$ e o valor obtido para $g$ é muito próximo do valor médio conhecido para a aceleração da gravidade na superfície da Terra, que é de aproximadamente $9,81 \mathrm{~m} / \mathrm{s}^{2}$, com desvio percentual de $1 \%$. Esse resultado assegura que o Tracker realizou o rastreamento de forma adequada e que as magnitudes dos parâmetros extraídos a partir da videoanálise estão em conformidade com os valores das grandezas físicas a eles associadas.

O resultado apresentado na Figura 5(a) mostra as medidas obtidas para a altura de lançamento $(n=0)$ e para as alturas máximas, $H_{n}$, atingidas após a enésima 


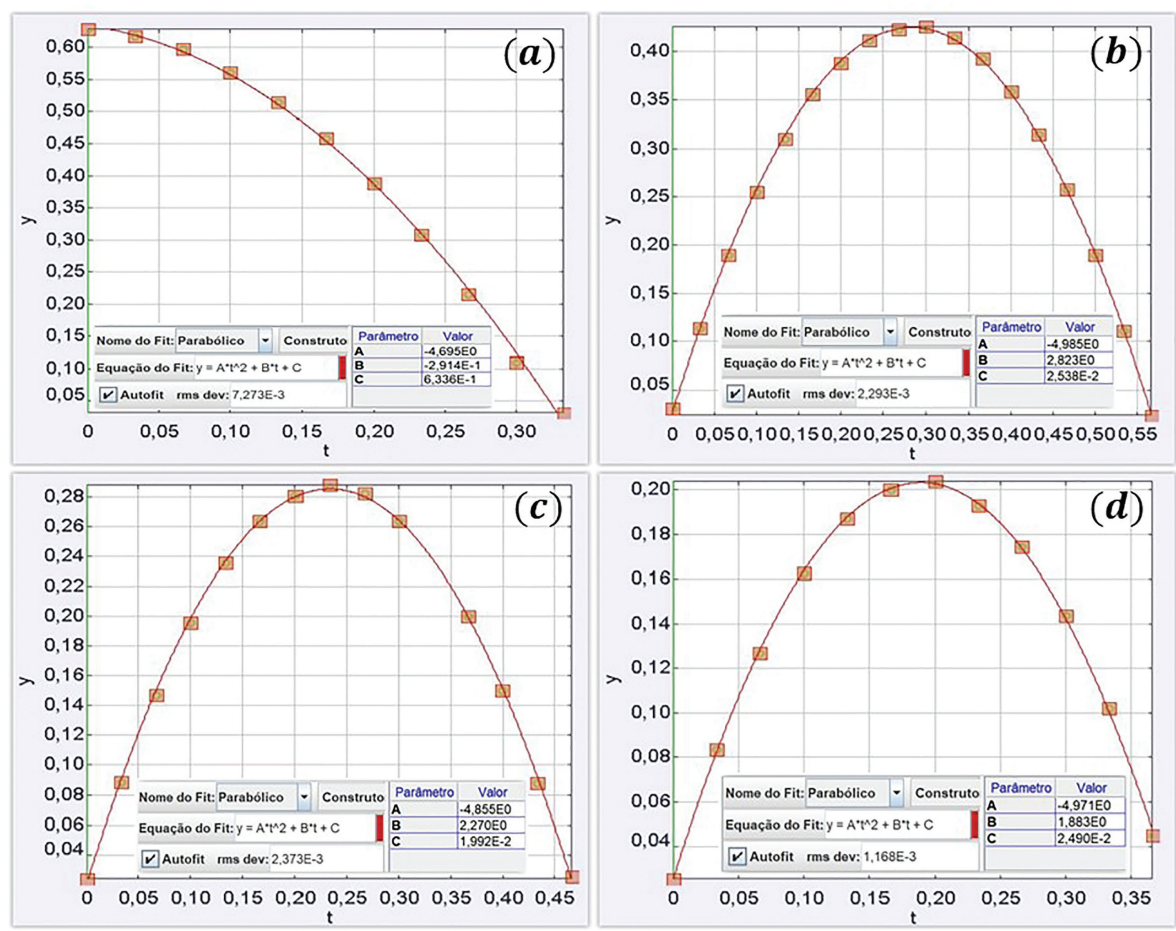

Figura 4: Coordenada vertical do centro de massa da esfera em função do tempo entre duas colisões sucessivas. Pontos: experimento. Curva vermelha: ajuste.

(a)

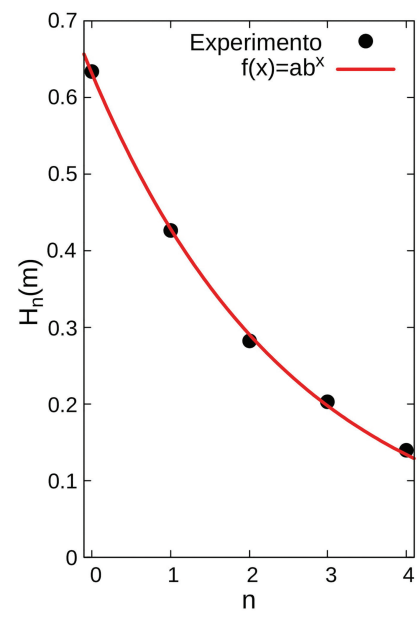

(b)

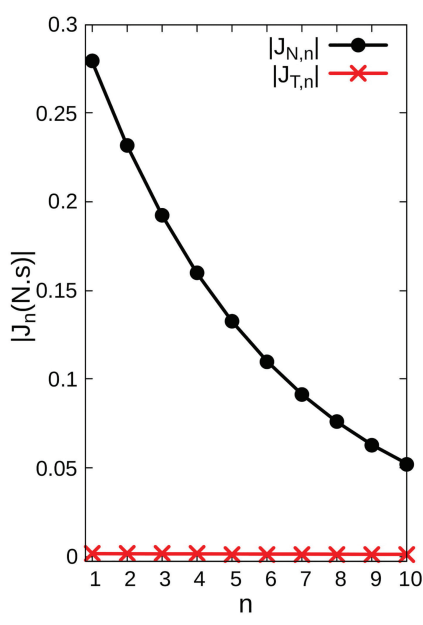

Figura 5: (a) Altura máxima atingida após a enésima colisão com o solo. Círculos: pontos experimentais. Curva sólida: ajuste a partir da função $f(x)=a b^{x}$, com $a=0,63108$ e $b=$ 0,679397. (b) Módulos dos impulsos normal $J_{N, n}$ (curva preta) e tangencial $J_{T, n}$ (curva vermelha) calculados em função de $n$.

colisão, sendo $n=1,2,3$ e 4 . O ajuste desses dados a partir da função $f(x)=a b^{x}$ encontra correspondência com a equação (4) através da identificação dos parâmetros $a$ e $b$ com a altura de lançamento $H_{0}$ e com o quadrado do coeficiente de restituição normal $e$, respectivamente. Os valores obtidos para essas grandezas a partir da análise gráfica foram $H_{0}=a=0,631 \mathrm{~m} \pm 0,006 \mathrm{~m}$,

Tabela 1: Tabela contendo a componente horizontal da velocidade após a enésima colisão, $v_{n, x}$, e a razão entre os valores consecutivos dessa velocidade, $\beta=v_{n, x} / v_{n-1, x}$, com $n \geq 1$, representando as medidas para o coeficiente de restituição tangencial.

\begin{tabular}{|c|c|c|}
\hline$n$ & $v_{n, x}(\mathrm{~m} / \mathrm{s})$ & \\
\hline 0 & $1,196 \pm 0,006$ & $\beta=v_{n, x} / v_{n-1, x}$ \\
\hline 1 & $1,166 \pm 0,007$ & $0,975 \pm 0,008$ \\
\hline 2 & $1,047 \pm 0,009$ & $0,898 \pm 0,009$ \\
\hline 3 & $1,08 \pm 0,02$ & $1,03 \pm 0,02$ \\
\hline 4 & $1,06 \pm 0,04$ & $0,98 \pm 0,04$ \\
\hline
\end{tabular}

medida entre o centro de massa da esfera e o piso do laboratório, e $e=\sqrt{b}=0,824 \pm 0,003$. Uma vez que a razão entre as alturas máximas sucessivas é igual ao quadrado do coeficiente de restituição $e^{2}$ (ver equação (2) ) e que a energia potencial é proporcional à altura do sólido, temos que essa energia, após cada colisão, preserva aproximadamente $68 \%$ do valor que possuía na colisão anterior. A função do coeficiente de restituição normal no estudo de colisões inelásticas bidimensionais, como as investigadas no experimento descrito neste artigo, é a de quantificar a perda e indicar a parcela da energia preservada após cada colisão. Essas perdas são atribuídas a dissipações associadas a deformações permanentes nas superfícies da esfera e do piso do laboratório.

Também realizamos um estudo de $v_{x}$ nos instantes imediatamente após as colisões, cujos resultados estão apresentados na segunda coluna da Tabela 11. com $v_{0, x}=1,195 \mathrm{~m} / \mathrm{s}\left(\delta v_{0, x}=0,006 \mathrm{~m} / \mathrm{s}\right)$ representando 
a velocidade da esfera no momento em que é lançada. Esses resultados foram obtidos pelo ajuste das medidas da coordenada $x$ do centro de massa da esfera em função do tempo nos intervalos temporais definidos entre o lançamento inicial e a primeira colisão e entre as colisões sucessivas, até a quarta colisão $(n=4)$. Em cada um desses intervalos a componente $x$ apresentou um comportamento linear típico do movimento uniforme, indicando a ausência de efeitos dissipativos, como o proveniente da resistência do ar. Os valores obtidos para a componente horizontal da velocidade após as colisões foram então, a partir da equação (5) e para $1 \leq n \leq 4$, utilizados para estimar os coeficientes de restituição tangenciais $\beta_{i}$ apresentados na terceira coluna da tabela. A partir desses valores obtivemos o valor $\beta=0,97 \pm 0,05$, com $\bar{\beta}=0,97$ correspondendo à média aritmética dos valores $\beta_{i}$ e a barra de erro $\delta \bar{\beta}=0,05$ estimada a partir do desvio padrão da distribuição correspondente. A apreciação do coeficiente de restituição tangencial $\beta$ a partir de seu valor médio se faz pertinente pelo fato desse valor se aproximar da unidade e, com isso, indicar a influência pequena da força de atrito na dinâmica das colisões, justificando o modelo teórico proposto nos livros didáticos. Não obstante, a análise de cada uma das colisões sucessivas a partir dos valores $\beta_{i}$ a elas associados acabou por revelar toda a riqueza associada à dinâmica desses eventos e que é atribuída ao movimento de rotação da esfera, conforme discutido a seguir.

O conjunto de valores obtidos para o coeficiente de restituição tangencial possui uma distribuição em que um desses valores supera ligeiramente a unidade, como observado para o coeficiente associado à terceira colisão $(n=3) \beta=1,03(\delta \beta=0,02)$ apresentado na Tabela 1 . sendo a incerteza $\delta \beta$ obtida a partir da expressão

$$
\delta \beta=\beta \sqrt{\frac{\delta v_{n, x}^{2}}{v_{n, x}^{2}}+\frac{\delta v_{n-1, x}^{2}}{v_{n-1, x}^{2}}},
$$

que descreve a propagação das incertezas associadas às medidas das componentes horizontais da velocidade.

Esse fato, que está associado ao aumento um tanto quanto inesperado da componente $v_{x}$ da velocidade do centro de massa da esfera após a colisão mencionada, pode ser entendido a partir das discussões apresentadas na referência [28, na qual esse mesmo fenômeno é relatado pelo autor ao analisar uma série de colisões sucessivas sofridas por uma "super ball", que, no caso, possui as mesmas características da esfera utilizada em nosso experimento. Esse comportamento pode ser entendido a partir da mudança de sentido da força de atrito, que às vezes aponta no mesmo sentido da componente horizontal da velocidade e, outras vezes, contra. Em particular, nas primeiras colisões, a velocidade angular de rotação, $\omega$, da esfera é relativamente baixa, de modo que a força de atrito se opõe à $v_{x}$ (ver Figura 3(b)), produzindo um torque que acaba por aumentar o valor de $\omega$. Esse cenário se repete nas colisões subsequentes até que $\omega$ atinge um valor crítico, a partir do qual, devido ao aumento da compressão do piso pela superfície da esfera no sentido contrário ao de $v_{x}$, a superfície do piso passa a reagir com uma força de atrito horizontal que aponta no sentido do movimento (da esquerda para a direita), favorecendo o movimento de translação em detrimento do movimento de rotação, quando observamos um aumento de $v_{x}$ acompanhado de uma redução da velocidade angular de rotação da esfera.

Voltando à análise do coeficiente de restituição tangencial $\beta$ a partir de seu valor médio $\bar{\beta}$ e considerando a expressão apresentada na equação $(9)$ para o impulso tangencial $J_{T}$, temos que o valor $\beta=0,97$ obtido, com desvio percentual de $3 \%$, resulta em um impulso tangencial $J_{T}$ muito pequeno, $J_{T} \approx 0$, o que, como mencionado anteriormente, corrobora com o resultado experimental apresentado na Figura 2(b), que descreve um comportamento aproximadamente linear para o deslocamento horizontal $x$ em função do tempo. O ínfimo impulso tangencial obtido em nosso experimento está associado à deformações de cisalhamento na direção tangencial à superfície, que são muito pequenas se comparadas às deformações na direção normal. Acreditamos que a magnitude dessas tensões de cisalhamento podem ser intensificadas em um cenário no qual tanto o coeficiente de atrito estático entre a esfera e a superfície quanto a velocidade do centro de massa da esfera no instante do lançamento assumam valores maiores. No caso, o aumento do coeficiente de atrito estático se faz necessário mediante o aumento da velocidade de lançamento, para que a esfera não deslize durante as colisões, o que acarretaria uma redução das deformações de cisalhamento promovidas pela força de atrito estático.

Na Figura 5(b) confrontamos os impulsos normal e tangencial, calculados, respectivamente, pelas equações (8) e (9) definidas com os parâmetros $g=9,7 \mathrm{~m} / \mathrm{s}^{2}$, $e=0,824, H_{0}=0,63 \mathrm{~m}, \beta=0,97, v_{0, x}=1,19 \mathrm{~m} / \mathrm{s} \mathrm{e}$ $m=0,044 \mathrm{~kg}$. Esse resultado evidencia o fato do impulso tangencial $J_{T}$, produzido pela força de atrito, ser mínimo se comparado ao impulso normal $J_{N}$ produzido pela força normal de contato, o que é coerente com o comportamento aproximadamente constante observado para a componente horizontal da velocidade da esfera e acaba por justificar de forma quantitativa o modelo de colisão apresentado na Figura 1 e considerado nos livros didáticos de física, que não leva em consideração a atuação da força de atrito.

\section{Considerações Finais}

Neste trabalho estudamos a dinâmica associada às colisões de uma esfera de borracha com a superfície do solo, após ser lançada de uma altura $H_{0}$ com velocidade horizontal $v_{0 x}$. A partir da videoanálise do movimento descrito por essa esfera, obtivemos valores para grandezas a ele associadas, como a altura de lançamento, as alturas máximas atingidas pela esfera 
após as colisões, as componentes horizontais e verticais das velocidades imediatamente após essas colisões, além da própria aceleração da gravidade local. Ainda, partindo do diagrama de forças apresentado na Figura 3(b), que adotamos como modelo para as colisões observadas, apresentamos um estudo com o objetivo de avaliar o papel desempenhado pelas forças que atuam sobre a esfera durante as colisões. Estudo esse que teve por base os valores obtidos para os coeficientes de restituição normal e tangencial, associados, respectivamente, à atuação das forças normal e de atrito aplicadas à esfera durante as colisões. No caso, os valores obtidos para os coeficientes de restituição tangencial $\beta$ foram próximos da unidade, indicando que a contribuição da força de atrito para o impulso fornecido à esfera durante as colisões é ínfima se comparada à da força normal, como pode ser observado pela dependência praticamente linear obtida para o deslocamento horizontal $x$ em função do tempo $t$ mostrada na Figura 2(b), que indica a invariância da componente horizontal da velocidade, $v_{x}$, e a ausência de forças contrárias ao movimento. A importância desse resultado está no fato dele justificar, quantitativa e experimentalmente, o modelo de colisão que é considerado com frequência nos livros didáticos para abordar o problema no qual um sólido com determinada massa colide de forma oblíqua contra uma superfície horizontal rígida, e que desconsidera a atuação da força de atrito. Quanto ao coeficiente de restituição normal $e$, obtivemos que ele estabelece uma relação entre as alturas de lançamento $H_{0}$ e da enésima colisão $H_{n}$ através da equação (4). Relação essa que foi considerada no ajuste dos dados experimentais que obtivemos para $H_{n}$, conforme mostrado na Figura 5(a). A partir desse ajuste foi extraído um valor médio de $e=0,824$ para as colisões consideradas $(n=1,2,3,4)$, que em seguida foi utilizado para estimar a fração da energia dissipada e o impulso normal $J_{N, n}$ fornecido à esfera durante as colisões, esta última grandeza representada pela curva preta na Figura 5(b), que mostra também, através da curva em vermelho, os resultados obtidos para o impulso tangencial $J_{T, n}$ associado a essas colisões, evidenciando quantitativamente o domínio praticamente absoluto da força normal de contato em comparação à força de atrito no que tange a influência dessas forças na dinâmica das colisões estudadas.

Como última consideração, destacamos o valor ligeiramente superior à unidade obtido para o coeficiente de restituição tangencial $\beta$ associado à terceira colisão, $\beta=$ 1,03 , indicando um aumento da componente horizontal da velocidade após esse evento. Esse resultado aparentemente inexpressivo sugere a existência de um cenário ainda mais rico associado à dinâmica das colisões produzidas em nosso experimento, no qual a força de atrito, que, de fato, como discutido, possui um efeito dissipativo praticamente desprezível, quantificado pelos ínfimos valores obtidos para $J_{T, n}$ apresentados na Figura 5(b), se revela importante devido a sua contribuição, como uma força não dissipativa, para o torque e, consequentemente, para a dinâmica de rotação da esfera. Essa conclusão se baseia na discussão apresentada na referência [28] e será explorada em trabalhos futuros nos quais pretendemos maximizar o efeito não dissipativo da força de atrito utilizando sólidos regulares de dimensões maiores, como esferas e discos, lançados de modo a colidirem com superfícies rígidas de diferentes rugosidades.

\section{Agradecimentos}

Os autores agradecem ao CEFET/RJ pela infraestrutura disponibilizada e pelo suporte financeiro através das bolsas de Iniciação Científica concedidas por meio do PROGRAMA INSTITUCIONAL DE BOLSAS DE INICIAÇÃO CIENTÍFICA - PIBIC 2019. Foram contemplados com as referidas bolsas R. S. Franco (Bolsista PIBIC) e V. S. Miranda (Bolsista PIBIC).

\section{REFERÊNCIAS}

[1] R. Stock, Physics Reports 135, 5 (1986).

[2] H.M. Nussenzveig, Curso de física básica: Mecânica (Editora Blucher, São Paulo, 2013), v. 1.

[3] D. Halliday, R. Resnick e J. Walker, Fundamentos de física: mecânica (LTC, Rio de Janeiro, 2009), v. 1, 8º ed.

[4] M. Alonso e E.J. Finn, Um curso universitário-Mecânica (Editora Blucher, São Paulo, 2018), v. 1.

[5] D.P. Meira Filho, J.K.S. Kamassury e R.C.S. Meira, Rev. Bras. Ensino Fís. 39, e4302 (2017).

[6] J.F. Nascimento Junior, V.E.S. Borges e R.M.M.F. Nascimento, Rev. Bras. Ensino Fís. 41, e20180219 (2019).

[7] F. Ramalho, N.G. Ferraro e P.A.T. Soares, Os fundamentos da física (Moderna, São Paulo, 2003), v. 1.

[8] P.A. Tipler e G. Mosca, Física para cientistas e engenheiros: mecânica, oscilações e ondas, termodinámica (Grupo Gen-LTC, Rio de Janeiro, 2000), v. 1.

[9] R.H. Doca, G.J. Biscuola e N.V. Boas, Os topicos da fisica 1: mecânica (Saraiva, São Paulo, 1983).

[10] A. Máximo e B. Alvarenga, Curso de física (Scipione, São Paulo, 2000) v. 1.

[11] H.D. Young e R.A Freedman, Física I (Pearson Educación, São Paulo, 2009).

[12] J.R. Bonjorno e C.M. Ramos, Física (FTD, São Paulo, 1992), v. 1.

[13] J.T. Jenkins, ASME. J. Appl. Mech. March 59, 120 (1992).

[14] https://physlets.org/tracker/

[15] N. Chanpichai e P. Wattanakasiwich, Thai. J. Phys. 5, 358 (2010).

[16] C. Sirisathitkul, P. Glawtanong, T. Eadkong e Y. Sirisathitkul, Rev. Bras. Ensino Fís. 35, 1504 (2013).

[17] P. Glawtanong, S. Ritphan, C. Sirisathitkul, C. Yaiprasert e Y. Sirisathitkul, Walailak J Sci \& Tech 8, 63 (2011).

[18] V.L.B. Jesus e D.G.G. Sasaki, Rev. Bras. Ensino Fís. 36, 3503 (2014).

[19] V.L.B. Jesus e D.G.G. Sasaki, Rev. Bras. Ensino Fís. 37, 1507 (2015). 
[20] A.V. Andrade-Neto, J.A. Cruz, M.S.R. Miltão e E.S. Ferreira, Rev. Bras. Ensino Fís. 35, 1 (2013).

[21] M.A. Pires e E.A. Veit, Rev. Bras. Ensino Fís. 28, 241 (2006).

[22] J.A. Lenz, N.C. Saavedra Filho e A.G. Bezerra Júnior, Abakós 2, 24 (2014).

[23] E.V. Rodrigues e D. Lavino, Rev. Bras. Ensino Fís. 42, e20190012 (2020).

[24] E.A. Lima, R.S. Dutra e P.V.S. Souza, Physics Education 55, 045021, (2020).

[25] L.O. Pereira, D.S.R. Ferreira, R.P. Freitas, A.R. Pimenta, V.S. Felix, E.A.S. Gonçalves e R.S. Dutra, Rev. Bras. Ensino Fís. 43, e20200498 (2021).

[26] S.F. Foerster, M.Y. Louge, H. Chang e K. Allia, Physics of Fluids 6, 1108 (1994).

[27] http://scidavis.sourceforge.net/

[28] R. Cross, Physics Education 55, 043001 (2020). 